\title{
BOURSES DE VOYAGE OFFERTES A DES JEUNES PAR L'ISA POUR SE RENDRE AU CONGRES MONDIAL D'ANDROLOGIE DE TOKYO (2 au 6 Mai 1993)
}

L'International Society of Andrology offre 20 bourses de voyage de 750 \$ US pour se rendre à Tokyo. Elles sont réservées à des scientifiques jeunes, avec une préférence pour ceux venant des pays en voie de développement, mais possibilité d'attribution à des européens ayant l'intention d'assister au congrès de Tokyo. La condition pour l'obtention de la bourse est la soumission d'un mini-poster pour le congrès. Les boursiers seront sélectionnés d'après la qualité scientifique de leur résumé. La date limite pour l'envoi des résumés est le 31 Décembre 1992.

Le premier auteur du mini-poster doit préciser son souhait de participer à la compétition pour l'obtention d'une bourse dans une lettre jointe au mini-poster et au formulaire d'inscription. Les bénéficiaires seront informés avant le congrès.

\section{RENOUVELLEMENT DU CONSEIL D'ADMINISTRATION}

Cinq des 15 membres du conseil devront être renouvelés à l'occasion du congrès de Nîmes. Les candidatures doivent être adressées au secrétaire général, le Docteur Jean BELAISCH, 36 rue de Tocqueville, 75017 PARIS.

\section{CONGRES DE NIMES}

Cinq prix de 5000 Frs seront offerts aux auteurs des posters à l'occasion du congrès de Nîmes, grâce à l'aide des laboratoires ALMED, IPSEN, ORGANON et SERONO, que nous remercions vivement. Deux seront décernés pour des travaux fondamentaux, 2 pour des travaux cliniques, 1 sera un "Prix de la SALF", décerné par l'assemblée du congrès. L'un des prix au moins sera réservé à une jeune équipe. Les bénéficiaires des prix pourront également présenter oralement leurs travaux, devant l'assemblée du congrès lors de la remise des prix, en 6 minutes, avec un maximum de 6 diapositives.

\section{LE MOT DU SECRETAIRE}

Les programmes d'enseignement de l'andrologie sont nécessairement variables selon les lieux où ils sont mis en oeuvre. Ils dépendent des compétences des organisateurs des enseignements et de leurs choix scientifiques. Neanmoins, une cohérence entre tous les enseignements est indispensable et la SALF s'est fixé pour but de faciliter les contacts entre les différents responsables nationaux afin que tous les diplômés d'andrologie de langue française aient un bagage commun minimum, à la condition du moins. qu'ils se soient donnés la peine d'assister à tous les cours ( et de les écouter !)

Cela n'empêche pas que les points de vue des andrologues puissent être très variés puisque, malgré des tendances nouvelles, la médecine demeure un art et non une science et que l'andrologie se veut une discipline médicale !..

Or, si l'andrologie doit pour beaucoup de spécialistes s'attacher avant tout à l'étude de la stérilité et de l'impuissance masculine, elle est aussi pour nombre d'andrologues dont Gabriel ARVIS a pris la tête, le lieu où l'on s'interroge sur le fait masculin, sur les attributs psychologiques de l'homme et sur son évolution dans le monde d'aujourd'hui.

Est-ce alors un hasard, mais on est bien contraint de constater que l'andrologie prend son essor alors que la position de l'homme est de plus en plus contestée et incertaine vis à vis de la femme..., et de la société.

Certes, ce n'est pas le cas sur la majeure partie du globe, et on ne peut déduire de ce qui est survenu dans les pays occidentaux que la même évolution ne tardera pas dans le reste du monde. Néanmoins, la transformation des positions respectives de l'homme et de la femme a été si rapide et si inattendue qu'on ne peut que s'interroger sur ses mécanismes.

A l'évidence, les machines à laver le linge et la vaisselle ont offert aux femmes un espace de temps qu'elles ont su utiliser pour entreprendre des travaux d'une autre sorte, et peut être plus encore, pour les envisager pour leurs propres filles. Pourtant, et bien que cela ait été discuté par tous les amis à qui j'en ai parlé, je me dernande si les machines dans leur ensemble, qui ont enlevé aux hommes leur supériorité puisque la force physique n'était plus un avantage, n'ont pas été la cause du "déclin" masculin! Et peut être, même, la petite machine à écrire, instrument banal de l'activite féminine, en permettant aux femmes d'avoir accès à toutes les règles des jeux du commerce et de l'industrie, a-t-elle accéléré la libération de la femme.

La place de l'homme est donc en voie de se réduire dans l'organisation sociale comme dans sa constellation familiale Les compromis entre hommes et femmes ne se décideront qu' après des discussions de plus en plus serrées.

Paraitra-t-il curieux de souhaiter que les andrologues ne soient pas les derniers à chercher les bases d'une véritable harmonie entre les sexes? 